Pacific

Journal of

Mathematics

\title{
ON THE GEOMETRY OF PRÜFER INTERSECTIONS OF VALUATION RINGS
}

\author{
BRUCE OLBERDING
}

Volume $273 \quad$ No. 2

February 2015 


\title{
ON THE GEOMETRY OF PRÜFER INTERSECTIONS OF VALUATION RINGS
}

\author{
BRUCE OLBERDING
}

\begin{abstract}
Let $F$ be a field, let $D$ be a subring of $F$ and let $Z$ be an irreducible subspace of the space of all valuation rings between $D$ and $F$ that have quotient field $F$. Then $Z$ is a locally ringed space whose ring of global sections is $A=\bigcap_{V \in Z} V$. All rings between $D$ and $F$ that are integrally closed in $F$ arise in such a way. Motivated by applications in areas such as multiplicative ideal theory and real algebraic geometry, a number of authors have formulated criteria for when $\boldsymbol{A}$ is a Prüfer domain. We give geometric criteria for when $A$ is a Prüfer domain that reduce this issue to questions of prime avoidance. These criteria, which unify and extend a variety of different results in the literature, are framed in terms of morphisms of $Z$ into the projective line $\mathbb{P}_{D}^{1}$.
\end{abstract}

\section{Introduction}

A subring $V$ of a field $F$ is a valuation ring of $F$ if for each nonzero $x \in F, x$ or $x^{-1}$ is in $V$; equivalently, the ideals of $V$ are linearly ordered by inclusion and $V$ has quotient field $F$. Although the ideal theory of valuation rings is straightforward, an intersection of valuation rings in $F$ can be quite complicated. Indeed, by a theorem of Krull [Matsumura 1980, Theorem 10.4], every integrally closed subring of $F$ is an intersection of valuation rings of $F$. In this article, we describe a geometrical approach to determining when an intersection $A$ of valuation rings of $F$ is a Prüfer domain, meaning that for each prime ideal $P$ of $A$, the localization $A_{P}$ is a valuation ring of $F$. Whether an intersection of valuation rings is Prüfer is of consequence in multiplicative ideal theory, where Prüfer domains are of central importance, and real algebraic geometry, where the real holomorphy ring is a Prüfer domain that expresses properties of fields involving sums of squares; see the discussion below. Over the past eighty years, Prüfer domains have been extensively studied from ideal-theoretic, homological and module-theoretic points of view; see, for example, [Fontana et al. 1997; Fuchs and Salce 2001; Gilmer 1968; Knebusch and Zhang 2002; Larsen and McCarthy 1971].

MSC2010: primary 13F05, 13F30; secondary 13B22, 14A15.

Keywords: Prüfer domain, valuation ring, Zariski-Riemann space. 
Throughout the paper $F$ denotes a field, $D$ is a subring of $F$ that need not have quotient field $F$, and $Z$ is a subspace of the Zariski-Riemann space $\mathfrak{X}$ of $F / D$, the space of all valuation rings of $F$ that contain $D$. The topology on $\mathfrak{X}$ is given by declaring the basic open sets to be those of the form $\left\{V \in \mathfrak{X}: t_{1}, \ldots, t_{n} \in V\right\}$, where $t_{1}, \ldots, t_{n} \in F$. We assume for technical convenience that $F \in Z$. With this notation fixed, the focus of this article is the holomorphy ring $^{1} A=\bigcap_{V \in Z} V$ of the subspace $Z$. Such a ring is integrally closed in $F$, and, as noted above, every ring between $D$ and $F$ that is integrally closed in $F$ occurs as the holomorphy ring of a subspace of $\mathfrak{X}$. In general it is difficult to determine the structure of $A$ from properties of $Z$, topological or otherwise; see [Olberding 2007; 2008; 2011], where the emphasis is on the case in which $D$ is a two-dimensional Noetherian domain with quotient field $F$. In this direction, there are a number of results that are concerned with when the holomorphy ring $A$ is a Prüfer domain with quotient field $F$. Geometrically, this is equivalent to $\operatorname{Spec}(A)$ being an affine scheme in $\mathfrak{X}$. Moreover, by virtue of the valuative criterion for properness, $A$ is a Prüfer domain with quotient field $F$ if and only if there are no nontrivial proper birational morphisms into the scheme $\operatorname{Spec}(A)$, an observation that motivates Temkin and Tyomkin's notion [2013] of Prüfer algebraic spaces.

We show in this article that the morphisms of $Z$ (viewed as a locally ringed space) into the projective line $\mathbb{P}_{D}^{1}$ determine whether the holomorphy ring $A$ of $Z$ is a Prüfer domain. A goal in doing so is to provide a unifying explanation for an interesting variety of results in the literature. By way of motivation, and because we will refer to them later, we recall these results here.

(1) Perhaps the earliest result in this direction is due to Nagata [1962, (11.11)]: When $Z$ is finite, the holomorphy ring $A$ of $Z$ is a Prüfer domain with quotient field $F$.

(2) Gilmer [1969, Theorem 2.2] shows that when $f$ is a nonconstant monic polynomial over $D$ having no root in $F$ and each valuation ring in $Z$ contains the set $S:=\{1 / f(t): t \in F\}$, then $A$ is a Prüfer domain with torsion Picard group and quotient field $F$. Rush [2001, Theorem 1.4] has since generalized this by allowing the polynomial $f$ to vary with the choice of $t$, but at the (necessary) expense of requiring the rational functions in $S$ to have certain numerators other than 1. Gilmer was motivated by a special case of this theorem due to Dress [1965], which states that when the field $F$ is formally real (meaning that -1 is not a sum of squares), then the subring of $F$ generated by $\left\{\left(1+t^{2}\right)^{-1}: t \in F\right\}$ is a Prüfer domain with quotient field $F$ whose set of valuation overrings is precisely the set of valuation rings of $F$

\footnotetext{
${ }^{1}$ This terminology is due to Roquette [1973, p. 362]. Viewing $Z$ as consisting of places rather than valuation rings, the elements of $A$ are precisely the elements of $F$ that have no poles (i.e., do not have value infinity) at the places in $Z$.
} 
for which -1 is not a square in the residue field. In the literature of real algebraic geometry, the Prüfer domain thus constructed is the real holomorphy ring of $F / D$. The fact that such rings are Prüfer has a number of interesting consequences for real algebraic geometry and sums of powers of elements of $F$; see, for example, [Becker 1982; Schülting 1982]. These rings are also the only known source of Prüfer domains having finitely generated ideals that cannot be generated by two elements, as was shown by Schülting [1979] and Swan [1984]; the related literature on this aspect of holomorphy rings is discussed in [Olberding and Roitman 2006]. The notion of existential closure leads to more general results on Prüfer holomorphy rings in function fields. For references on this generalization, see [Olberding 2006].

(3) Roquette [1973, Theorem 1] proves that when there exists a nonconstant monic polynomial $f \in A[T]$ which has no root in the residue field of $V$ for each valuation ring $V \in Z$ (i.e., the residue fields are "uniformly algebraically non-closed"), $A$ is a Prüfer domain with torsion Picard group and quotient field $F$. Roquette [1973, p. 362] developed these ideas as a general explanation for his principal ideal theorem, which states that the ring of totally $p$-integral elements of a formally $p$-adic field is a Bézout domain; that is, every finitely generated ideal is principal. In particular, if there is a bound on the size of the residue fields of the valuation rings in $Z$, then $A$ is a Bézout domain [Roquette 1973, Theorem 3]. Motivated by just such a situation, Loper [1994] independently proved similar results in order to apply them to the ring of integer-valued polynomials of a domain $R$ with quotient field $F: \operatorname{Int}(R)=\{g(T) \in F[T]: g(R) \subseteq R\}$.

(4) In [Olberding and Roitman 2006] it is shown that if the holomorphy ring $A$ of $Z$ contains a field of cardinality greater than that of $Z$, then $A$ is a Bézout domain.

In this article we offer a geometric explanation for these results that reduces all the arguments to a question of homogeneous prime avoidance in the projective line $\mathbb{P}_{D}^{1}:=\operatorname{Proj}\left(D\left[T_{0}, T_{1}\right]\right)$. Nagata's theorem in (1) reduces to the observation that a finite set of points of $\mathbb{P}_{D}^{1}$ is contained in an affine open subset of $\mathbb{P}_{D}^{1}$. The example in (4) is explained similarly by showing that a "small" enough set of points in $\mathbb{P}_{D}^{1}$ is contained in an affine open set. And finally, in cases (2) and (3), the condition on the residue fields guarantees that the image of each $D$-morphism $Z \rightarrow \mathbb{P}_{D}^{1}$ is contained in the open affine subset $\left(\mathbb{P}_{D}^{1}\right)_{g}$, where $g$ is the homogenization of $f$.

To frame things geometrically, we view $Z$ as a locally ringed space. Its structure sheaf $\mathrm{O}_{Z}$ is defined for each nonempty open subset $U$ of $Z$ by $\mathrm{O}_{Z}(U)=\bigcap_{V \in Z} V$, while the ring of sections of the empty set is defined to be the trivial ring with $0=1$; thus $\mathrm{O}_{Z}$ is the holomorphy sheaf of $Z$. The restriction maps on $\mathrm{O}_{Z}$ off the empty set are simply set inclusion, and the stalks of $\mathrm{O}_{Z}$ are the valuation rings in $Z$. The standing assumption that $F$ is one of the valuation rings in $Z$ guarantees that $Z$ is an irreducible space; irreducibility in turn guarantees that $\mathrm{O}_{Z}$ is a sheaf. (Note 
that since we are interested in the ring $A=\bigcap_{V \in Z} V$, the assumption $F \in Z$ is no limitation.) When considering irreducible subspaces $Y$ of $\mathfrak{X}$, we similarly treat $Y$ as a locally ringed space with structure sheaf defined in this way.

By a morphism we always mean a morphism in the category of locally ringed spaces. If $X$ and $Y$ are locally ringed spaces with fixed morphisms $\alpha: X \rightarrow \operatorname{Spec}(D)$ and $\beta: Y \rightarrow \operatorname{Spec}(D)$, then a morphism $\phi: X \rightarrow Y$ is a $D$-morphism if $\alpha=\beta \circ \phi$. A scheme $X$ is a $D$-scheme if a morphism $\phi: X \rightarrow \operatorname{Spec}(D)$ is fixed. There is a morphism $\delta=\left(d, d^{\#}\right): Z \rightarrow \operatorname{Spec}(D)$ defined by letting $d$ be the continuous map that sends a valuation ring in $Z$ to its center in $D$, and by letting $d^{\#}: \mathrm{O}_{\mathrm{Spec}(D)} \rightarrow d_{*} \mathrm{O}_{Z}$ be the sheaf morphism defined for each open subset $U$ of $\operatorname{Spec}(D)$ by the set inclusion $d_{U}^{\#}: 0_{\operatorname{Spec}(D)}(U) \rightarrow \mathrm{O}_{Z}\left(d^{-1}(U)\right)$. Thus when considering $D$-morphisms from $Z$ to $X$, with $X$ a $D$-scheme, we always assume that the structure morphism $Z \rightarrow \operatorname{Spec}(D)$ is the one defined above.

\section{Morphisms into projective space}

In this section we describe the $D$-morphisms of $Z$ into projective space by proving an analogue of the fact that morphisms from schemes into projective space are determined by invertible sheaves. Our main technical device in describing such morphisms is the notion of a projective model, as defined in [Zariski and Samuel 1975, Chapter VI, §17]. Let $t_{0}, \ldots, t_{n}$ be nonzero elements of $F$, and for each $i=0,1, \ldots, n$, define $D_{i}=D\left[t_{0} / t_{i}, \ldots, t_{n} / t_{i}\right]$ and $U_{i}=\operatorname{Spec}\left(D_{i}\right)$. Then the projective model of $F / D$ defined by $t_{0}, \ldots, t_{n}$ is

$$
X=\left\{\left(D_{i}\right)_{P}: P \in \operatorname{Spec}\left(D_{i}\right), i=0,1, \ldots, n\right\} .
$$

The projective model $X$ is a topological space whose basic open sets are of the form $\left\{R \in X: u_{0}, \ldots, u_{m} \in R\right\}$, where $u_{0}, \ldots, u_{m} \in F$, and which is covered by the open subsets $\left\{\left(D_{i}\right)_{P}: P \in U_{i}\right\}, i=0,1, \ldots, n$. Define a sheaf $\mathscr{O}_{X}$ of rings on $X$ for each nonempty open subset $U$ of $X$ by $0_{X}(U)=\bigcap_{R \in U} R$, and let the ring of sections of the empty set be the trivial ring with $0=1$. Since $X$ is irreducible, $O_{X}$ is a sheaf and hence $\left(X, O_{X}\right)$ is a scheme, and in light of the following remark, it is a projective scheme.

Remark 2.1. If $X$ is a projective model defined by $n+1$ elements, then there is a closed immersion $X \rightarrow \mathbb{P}_{D}^{n}$. For let $X$ be the projective model defined by $t_{0}, \ldots, t_{n} \in F$. For each $i=0,1, \ldots, n$, let $b_{i}: D\left[T_{0} / T_{i}, \ldots, T_{n} / T_{i}\right] \rightarrow D_{i}$ be the $D$-algebra homomorphism that sends $T_{j} / T_{i}$ to $t_{j} / t_{i}$, and let

$$
a_{i}: \operatorname{Spec}\left(D_{i}\right) \rightarrow \operatorname{Spec}\left(D\left[T_{0} / T_{i}, \ldots, T_{n} / T_{i}\right]\right)
$$

be the induced continuous map of topological spaces. Then the scheme morphisms $\left(a_{i}, b_{i}\right): \operatorname{Spec}\left(D_{i}\right) \rightarrow \operatorname{Spec}\left(D\left[T_{0} / T_{i}, \ldots, T_{n} / T_{i}\right]\right)$ glue together to a morphism 
$\phi: X \rightarrow \mathbb{P}_{D}^{n}$ [Hartshorne 1977, p. 88], which, by virtue of the way it is constructed, is a closed immersion [de Jong et al. 2005-, Lemma 01QO].

Let $t_{0}, \ldots, t_{n}$ be nonzero elements of $F$, and let $X$ be the projective model of $F / D$ defined by $t_{0}, \ldots, t_{n}$. For each valuation $\operatorname{ring} V$ in $Z$, there exists $i=0,1, \ldots, n$ such that $t_{j} / t_{i} \in V$ for all $j$, and it follows that each valuation ring $V$ in $Z$ dominates a unique local ring $R$ in the model $X$, meaning that $R \subseteq V$ and the maximal ideal of $R$ is contained in the maximal ideal of $V$. The domination morphism $\delta=\left(d, d^{\#}\right): Z \rightarrow X$ is defined by letting $d$ be the continuous map that sends a valuation ring in $Z$ to the local ring in $X$ that it dominates, and by letting $d^{\#}: \mathrm{O}_{X} \rightarrow d_{*} \mathrm{O}_{Z}$ be the sheaf morphism defined for each open subset $U$ of $Z$ by the set inclusion $d_{U}^{\#}: \mathrm{O}_{X}(U) \rightarrow \mathrm{O}_{Z}\left(d^{-1}(U)\right)$.

Let $\gamma: X \rightarrow \mathbb{P}_{D}^{n}$ be the closed immersion defined in Remark 2.1, and let $\delta: Z \rightarrow X$ denote the domination morphism. Then we say that the $D$-morphism $\gamma \circ \delta$ is the morphism defined by $t_{0}, \ldots, t_{n}$. We show in Proposition 2.3 that each $D$-morphism $Z \rightarrow \mathbb{P}_{D}^{n}$ arises in this way. Our standing assumption $F \in Z$ is used in a strong way here, in that the proposition relies on a lemma which shows that the $D$-morphisms from $Z$ into projective space are calibrated by the inclusion morphism $\operatorname{Spec}(F) \rightarrow Z$.

Lemma 2.2. Let $\iota: \operatorname{Spec}(F) \rightarrow Z$ be the canonical morphism, let $\phi=\left(f, f^{\#}\right)$ : $Z \rightarrow X$ and $\gamma=\left(g, g^{\#}\right): Z \rightarrow X$ be morphisms of locally ringed spaces, where $X$ is a separated scheme, and let $\eta=f(F)$. Then $\phi=\gamma$ if and only if $\phi \circ \iota=\gamma \circ \iota$ if and only if $\eta=f(F)=g(F)$ and $f_{\eta}^{\#}=g_{\eta}^{\#}$.

Proof. Suppose that $\eta=f(F)=g(F)$ and $f_{\eta}^{\#}=g_{\eta}^{\#}$. Let $U$ be an affine open subset of $X$ containing $\eta$, and let $Y=f^{-1}(U)$. Then $Y$ is a locally ringed space with structure sheaf $O_{Y}$ defined for each open set $W$ in $Y$ by $O_{Y}(W)=O_{Z}(W)$. We claim that $\left.\phi\right|_{Y}=\left.\gamma\right|_{Y}$. Since $U$ is affine and $Y$ is a locally ringed space, the morphisms $\left.\phi\right|_{Y}$ and $\left.\gamma\right|_{Y}$ are equal if and only if $f_{U}^{\#}=g_{U}^{\#}$ [Holme 2012, Theorem 10.8 , p. 200]. Now since $\mathrm{O}_{Z}(Y) \subseteq \mathrm{O}_{Z, F}=F$ and the restriction maps on the sheaf $\mathrm{O}_{Z}$ are set inclusions, for each $s \in \mathcal{O}_{X}(U)$ we have $f_{U}^{\#}(s)=f_{\eta}^{\#}(s)=g_{\eta}^{\#}(s)=g_{U}^{\#}(s)$. Thus $f_{U}^{\#}=g_{U}^{\#}$, and hence $\left.\phi\right|_{Y}=\left.\gamma\right|_{Y}$. Finally, let $\left\{U_{i}\right\}$ be the collection of all affine open subsets of $X$ that contain $\eta$. Then $\left\{f^{-1}\left(U_{i}\right)\right\}$ is a cover of $Z$, and we have shown that $\phi$ and $\gamma$ restrict to the same morphism on each of these open sets, so we conclude that $\phi=\gamma$. It is straightforward to verify that $\phi \circ \iota=\gamma \circ \iota$ if and only if $f(F)=g(F)$ and $f_{\eta}^{\#}=g_{\eta}^{\#}$, so the lemma follows.

Proposition 2.3. If $\phi: Z \rightarrow \mathbb{P}_{D}^{n}$ is a D-morphism, then there exist $t_{0}, \ldots, t_{n} \in F$ such that $\phi$ is defined by $t_{0}, \ldots, t_{n}$.

Proof. Write $\phi=\left(f, f^{\#}\right)$, let $\eta=f(F)$, and let $S=\mathbb{P}_{D}^{n}=\operatorname{Proj}\left(D\left[T_{0}, \ldots, T_{n}\right]\right)$. For each $i=0, \ldots, n$, let $U_{i}$ be the open affine set $S_{T_{i}}$, so that $S=U_{0} \cup \cdots \cup U_{n}$. Let 
$\alpha=\left(a, a^{\#}\right): \operatorname{Spec}(F) \rightarrow S$ be the composition of $\phi$ with the canonical morphism $\operatorname{Spec}(F) \rightarrow Z$, and note that for each $i$, we have $a_{U_{i}}^{\#}(s)=f_{S, \eta}^{\#}(s)$ for all $s \in$ $\mathrm{O}_{S}\left(U_{i}\right)$. Since $\alpha$ is a morphism of schemes into projective $n$-space over $D$, there exist $t_{0}, \ldots, t_{n} \in F$ such that $f_{U_{i}}^{\#}\left(T_{j} / T_{i}\right)=t_{j} / t_{i}$ for each $i, j$; see the proof of Theorem II.7.1 of [Hartshorne 1977, p. 150]. Let $X$ be the projective model of $F / D$ defined by $t_{0}, \ldots, t_{n}$. Then $t_{0}, \ldots, t_{n}$ can be viewed as global sections of an invertible sheaf on $X$ that is the image of the twisting sheaf $O(1)$ of $S$. By the theorem just cited and its proof, there is then a unique $D$-morphism $\gamma=\left(g, g^{\#}\right): X \rightarrow S$ such that $g_{U}^{\#}=f_{U}^{\#}$ for each open set $U$ of $S$ and $g: X \rightarrow S$ is the continuous map that for each $i=0, \ldots, n$ sends the equivalence class of a prime ideal $P$ in $\operatorname{Spec}\left(D\left[t_{0} / t_{i}, \ldots, t_{n} / t_{i}\right]\right) \subseteq X$ to the equivalence class of the prime ideal $\left(f_{U_{i}}^{\#}\right)^{-1}(P)$ in $U_{i}=\operatorname{Spec}\left(D\left[T_{0} / T_{i}, \ldots, T_{n} / T_{i}\right]\right)$. Then, with $\delta=\left(d, d^{\#}\right): Z \rightarrow X$ the domination morphism, $\gamma \circ \delta: Z \rightarrow S$ is a $D$-morphism. Moreover, $g(d(F))=g(F)=\eta=f(F)$ and (viewing $F$ as a point in both $X$ and $Z),\left(d^{\#} \circ g^{\#}\right)_{F}=d_{F}^{\#} \circ g_{\eta}^{\#}=f_{\eta}^{\#}$. Therefore, by Lemma 2.2, $\phi=\gamma \circ \delta$.

Corollary 2.4. Every D-morphism $\phi: Z \rightarrow \mathbb{P}_{D}^{n}$ lifts to a unique D-morphism $\widetilde{\phi}: \mathfrak{X} \rightarrow \mathbb{P}_{D}^{n}$.

Proof. Let $\phi: Z \rightarrow \mathbb{P}_{D}^{n}$ be a $D$-morphism. Then by Proposition 2.3, there exist a projective model $X$ of $F / D$ and a $D$-morphism $\gamma: X \rightarrow \mathbb{P}_{D}^{n}$ such that $\phi=\left.\gamma \circ \delta\right|_{Z}$, where $\delta: Z \rightarrow X$ is the domination map. Since $X$ is a projective model of $F / D$, each valuation ring in $\mathfrak{X}$ dominates $X$, and hence $\delta: Z \rightarrow X$ extends to the domination morphism $\widetilde{\delta}: \mathfrak{X} \rightarrow X$. Thus $\widetilde{\phi}=\gamma \circ \widetilde{\delta}$ lifts $\phi$. If there is another morphism $\psi: \mathfrak{X} \rightarrow \mathbb{P}_{D}^{n}$ that lifts $\phi$, then with $\iota: \operatorname{Spec}(F) \rightarrow Z$ the canonical morphism, $\psi \circ \iota=\phi \circ \iota=\widetilde{\phi} \circ \iota$, so that by Lemma $2.2, \psi=\widetilde{\phi}$.

Remark 2.5. By Lemma 2.2, the $D$-morphisms $Z \rightarrow \mathbb{P}_{D}^{n}$ are determined by their composition with the morphism $\operatorname{Spec}(F) \rightarrow \mathbb{P}_{D}^{n}$. Conversely, by Corollary 2.4, each $D$-morphism $\operatorname{Spec}(F) \rightarrow Z$ lifts to a unique morphism $Z \rightarrow \mathfrak{X}$. Thus the $D$-morphisms $Z \rightarrow \mathbb{P}_{D}^{n}$ are in one-to-one correspondence with the $F$-valued points of $\mathbb{P}_{D}^{n}$.

\section{A geometrical characterization of Prüfer domains}

We show in this section that if $Z$ has the property that the image of every $D$ morphism $Z \rightarrow \mathbb{P}_{D}^{1}$ of locally ringed spaces factors through an affine scheme, then the holomorphy ring $A$ of $Z$ is a Prüfer domain. A special case in which this is satisfied is when there is a homogeneous polynomial $f\left(T_{0}, T_{1}\right)$ of positive degree $d$ such that the image of each such morphism is contained in $\left(\mathbb{P}_{D}^{1}\right)_{f}$. In this case, we show that the Prüfer domain $A$ has torsion Picard group. 
Theorem 3.1. The ring $A=\bigcap_{V \in Z} V$ is a Prüfer domain with quotient field $F$ if and only if every $D$-morphism $Z \rightarrow \mathbb{P}_{D}^{1}$ factors through an affine scheme.

Proof. Suppose $A$ is a Prüfer domain, and let $\phi: Z \rightarrow \mathbb{P}_{D}^{1}$ be a $D$-morphism. By Proposition 2.3, there exist a projective model $X$ of $F / D$ and a $D$-morphism $\gamma: X \rightarrow \mathbb{P}_{D}^{1}$ such that $\phi=\gamma \circ \delta$, where $\delta: Z \rightarrow X$ is the domination morphism. Since $A$ is a Prüfer domain with quotient field $F$, every localization of $A$ is a valuation domain and hence dominates a local ring in $X$. Since every valuation ring in $Z$ contains $A$, it follows that $\phi$ factors through the affine scheme $\operatorname{Spec}(A)$.

Conversely, suppose that every $D$-morphism $Z \rightarrow \mathbb{P}_{D}^{1}$ factors through an affine scheme. Let $P$ be a prime ideal of $A$. To prove that $A_{P}$ is a valuation domain with quotient field $F$, it suffices to show that for each $0 \neq t \in F$ we have $t \in A_{P}$ or $t^{-1} \in A_{P}$. Let $0 \neq t \in F$, and let $X$ be the projective model of $F / D$ defined by $1, t$. Then by Remark 2.1 , there is a closed immersion of $X$ into $\mathbb{P}_{D}^{1}$. Let $\phi=\left(f, f^{\#}\right): Z \rightarrow \mathbb{P}_{D}^{1}$ be the $D$-morphism that results from composing this closed immersion with the domination morphism $Z \rightarrow X$. In particular, with $v=f(F)$, we have $f_{v}^{\#}\left(T_{1} / T_{0}\right)=t$ and $f_{v}^{\#}\left(T_{0} / T_{1}\right)=t^{-1}$.

By assumption, there are a ring $R$ and $D$-morphisms $\delta=\left(d, d^{\#}\right): Z \rightarrow \operatorname{Spec}(R)$ and $\gamma=\left(g, g^{\#}\right): \operatorname{Spec}(R) \rightarrow \mathbb{P}_{D}^{1}$ such that $\phi=\gamma \circ \delta$. By replacing $R$ with its image in $F$ under $d_{\eta}^{\#}$, where $\eta=d(F)$, we may assume by Lemma 2.2 that $R$ is a subring of $F$ and that $\delta$ is the domination morphism. Then since $R$ is the ring of global sections of $\operatorname{Spec}(R)$ and $A$ is the ring of global sections of $Z$, it follows that $R \subseteq A$, and hence $Q=R \cap P$ is a prime ideal of $R$. Let $x=g(Q)$. Then $x \in\left(\mathbb{P}_{D}^{1}\right)_{T_{0}}$ or $x \in\left(\mathbb{P}_{D}^{1}\right)_{T_{1}}$. In the former case, $f_{x}^{\#}\left(T_{1} / T_{0}\right)=t$, and in the latter, $f_{x}^{\#}\left(T_{0} / T_{1}\right)=t^{-1}$. But $f^{\#}=d^{\#} \circ g^{\#}$ and $d^{\#}$ restricts on each nonempty open subset of $\operatorname{Spec}(R)$ to the inclusion mapping, so either $x \in\left(\mathbb{P}_{D}^{1}\right)_{T_{0}}$, so that $t=f_{x}^{\#}\left(T_{1} / T_{0}\right)=g_{x}^{\#}\left(T_{1} / T_{0}\right) \in R_{Q} \subseteq A_{P}$, or $x \in\left(\mathbb{P}_{D}^{1}\right)_{T_{1}}$, so that $t^{-1}=f_{x}^{\#}\left(T_{0} / T_{1}\right)=g_{x}^{\#}\left(T_{0} / T_{1}\right) \in R_{Q} \subseteq A_{P}$. This proves that $A$ is a Prüfer domain with quotient field $F$.

Nagata's theorem discussed in (1) follows then from prime avoidance:

Corollary 3.2 [Nagata 1962, (11.11)]. If $Z$ is a finite set, then $A$ is a Prüfer domain with quotient field $F$.

Proof. Let $\phi: Z \rightarrow \mathbb{P}_{D}^{1}$ be a $D$-morphism. Then the image of $\phi$ in $\mathbb{P}_{D}^{1}$ is finite, so by homogeneous prime avoidance [Bruns and Herzog 1993, Lemma 1.5.10], there exists a homogeneous polynomial $f$ (necessarily of positive degree) in the irrelevant ideal $\left(T_{0}, T_{1}\right)$ of $D\left[T_{0}, T_{1}\right]$ such that $f$ is not in the union of the finitely many homogeneous prime ideals corresponding to the image of $Z$ in $\mathbb{P}_{D}^{1}$; i.e., the image of $\phi$ is contained in $\left(\mathbb{P}_{D}^{1}\right)_{f}$. This subset is affine [Eisenbud and Harris 2000, Exercise III.10, p. 99], so by Theorem 3.1, $A$ is a Prüfer domain with quotient field $F$. 
In fact, when $Z$ is finite, $A$ is a Bézout domain: If $M$ is a maximal ideal of $A$, then $A_{M}$ is a valuation domain, but since $Z$ is finite, $A_{M}=\bigcap_{V \in Z} V A_{M}$, which, since $A_{M}$ is a valuation domain, forces $A_{M}=V$ for some $V \in Z$. Therefore, $A$ has only finitely many maximal ideals, so that every invertible ideal is principal, and hence $A$ is a Bézout domain.

In Theorem 3.5, we give a criterion for when $A$ is a Prüfer domain with torsion Picard group. In this case, each $D$-morphism $Z \rightarrow \mathbb{P}_{D}^{1}$ not only factors through an affine scheme, but has image in an affine open subscheme of $\mathbb{P}_{D}^{1}$. For lack of a precise reference, we note the following standard observation.

Lemma 3.3. Let $X$ be a projective model of $F / D$ defined by $t_{0}, \ldots, t_{n} \in F$, and let $f\left(T_{0}, \ldots, T_{n}\right) \in D\left[T_{0}, \ldots, T_{n}\right]$ be homogeneous of positive degree $d$ such that $f\left(t_{0}, \ldots, t_{n}\right) \neq 0$. Let

$$
R=\{0\} \cup\left\{\frac{h\left(t_{0}, \ldots, t_{n}\right)}{f\left(t_{0}, \ldots, t_{n}\right)^{e}}: e \geq 0 \text { and } h \text { is a homogeneous form of degree de }\right\} .
$$

Then $\left\{R_{P}: P \in \operatorname{Spec}(R)\right\}$ is an open affine subset of $X$.

Proof. Let $S=\mathbb{P}_{D}^{n}$. Then $S_{f}$ is an open affine subset of $S$ [Eisenbud and Harris 2000, Exercise III.10, p. 99]. By Remark 2.1, there is a closed immersion $\gamma=$ $\left(g, g^{\#}\right): X \rightarrow S$ such that with $\eta=g(F)$, we have $g_{\eta}^{\#}\left(T_{j} / T_{i}\right)=t_{j} / t_{i}$ for each $i, j$. Since $S_{f}$ is an open affine subset of $S$ and $\gamma$ is a closed immersion, $g^{-1}\left(S_{f}\right)$ is an open affine subset of $X$ whose ring of sections is $g_{\eta}^{\#}\left(\mathcal{O}_{S}\left(S_{f}\right)\right)$ [de Jong et al. 2005-, Lemma 01IN]. Now $\mathcal{O}_{S}\left(S_{f}\right)$ is the ring consisting of 0 and the rational functions of the form $h / f^{e}$, where $e>0$ and $h$ is a homogeneous form of degree $d e$. Moreover, for such a rational function, since $f\left(t_{0}, \ldots, t_{n}\right) \neq 0$, we have that $f\left(T_{0}, \ldots, T_{n}\right)$ is a unit in $\mathrm{O}_{S, \eta}$ and

$$
g_{\eta}^{\#}\left(\frac{h\left(T_{0}, \ldots, T_{n}\right)}{f\left(T_{0}, \ldots, T_{n}\right)^{e}}\right)=\frac{h\left(t_{0}, \ldots, t_{n}\right)}{f\left(t_{0}, \ldots, t_{n}\right)^{e}} \in R .
$$

Thus $g_{\eta}^{\#}\left(\mathcal{O}_{S}\left(S_{f}\right)\right)=R$, which proves the lemma.

Lemma 3.4. Let $t_{0}, t_{1}, \ldots, t_{n}$ be nonzero elements of $F$, and let $f$ be a homogeneous polynomial in $D\left[T_{0}, \ldots, T_{n}\right]$ of positive degree $d$. Then the following are equivalent.

(1) $t_{0}^{d}, \ldots, t_{n}^{d} \in f\left(t_{0}, \ldots, t_{n}\right) A$.

(2) $\left(t_{0}, \ldots, t_{n}\right)^{d} A=f\left(t_{0}, \ldots, t_{n}\right) A$.

(3) The image of the morphism $Z \rightarrow \mathbb{P}_{D}^{n}$ defined by $t_{0}, \ldots, t_{n}$ is in $\left(\mathbb{P}_{D}^{n}\right)_{f}$.

Proof. Let $u=f\left(t_{0}, \ldots, t_{n}\right)$. First we claim that (1) implies (2). If $V \in Z$, then there is $i$ such that $t_{i}$ divides in $V$ each of $t_{0}, \ldots, t_{n}$. It follows that when $\sum_{i} e_{i}=d$ for 
nonnegative integers $e_{i}$, we have $t_{0}^{e_{0}} t_{1}^{e_{1}} \ldots t_{n}^{e_{n}} \in t_{i}^{d} V$. Thus by (1), $t_{0}^{e_{0}} t_{1}^{e_{1}} \ldots t_{n}^{e_{n}} \in u V$, so that $t_{0}^{e_{0}} t_{1}^{e_{1}} \ldots t_{n}^{e_{n}} \in u A$. Statement (2) now follows.

To see that (2) implies (3), let $\gamma=\left(g, g^{\#}\right): Z \rightarrow \mathbb{P}_{D}^{n}$ be the morphism defined by $t_{0}, \ldots, t_{n}$. By $(2), u=f\left(t_{0}, \ldots, t_{n}\right)$ is nonzero. Define

$$
\begin{aligned}
& R=\{0\} \cup\left\{\frac{h\left(t_{0}, \ldots, t_{n}\right)}{u^{e}}: e \geq 0 \text { and } h \text { is a homogeneous form of degree } d e\right\}, \\
& S=\{0\} \cup\left\{\frac{h\left(T_{0}, \ldots, T_{n}\right)}{f\left(T_{0}, \ldots, T_{n}\right)^{e}}: e \geq 0 \text { and } h \text { is a homogeneous form of degree } d e\right\},
\end{aligned}
$$

so that $\left(\mathbb{P}_{D}^{n}\right)_{f}=\operatorname{Spec}(S)$. Let $\alpha=\left(a, a^{\#}\right): \operatorname{Spec}(R) \rightarrow \operatorname{Spec}(S)$ be the morphism induced by the ring homomorphism $a^{\#}: S \rightarrow R$ given by evaluation at $t_{0}, \ldots, t_{n}$. Observe that $R \subseteq A$, since if $h$ is a homogeneous form in $D\left[T_{0}, \ldots, T_{n}\right]$ of degree $d e$, then by (2), $h\left(t_{0}, \ldots, t_{n}\right) \in\left(t_{0}, \ldots, t_{n}\right)^{d e} A=u^{e} A$, so that $R \subseteq A$. Now let $\beta: Z \rightarrow$ $\operatorname{Spec}(R)$ be the induced domination morphism. We claim that $\gamma=\alpha \circ \beta$. Indeed, by Lemma 3.3, $\operatorname{Spec}(R)$ is an affine submodel of the projective model $X$ of $F / D$ defined by $t_{0}, \ldots, t_{n}$, and $\gamma$ factors through $X$. Since $\beta$ is the domination mapping, it follows that $\gamma=\alpha \circ \beta$, and hence the image of $\gamma$ is contained in $\operatorname{Spec}(S)=\left(\mathbb{P}_{D}^{n}\right)_{f}$.

Finally, to see that (3) implies (1), let $U=\left(\mathbb{P}_{D}^{n}\right)_{f}$ and let $\gamma=\left(g, g^{\#}\right): Z \rightarrow \mathbb{P}_{D}^{n}$ be the morphism defined by $t_{0}, \ldots, t_{n}$. By (3), $Z \subseteq g^{-1}(U)$, so $S$, the ring of sections of $U$, is mapped via $g_{U}^{\#}$ into the holomorphy ring $A$ of $Z$. But the image of $g_{U}^{\#}$ is $R$, so $R \subseteq A$, and hence every element of $F$ of the form $t_{i}^{d} / u$ is an element of $A$, from which (1) follows.

Theorem 3.5. The ring $A=\bigcap_{V \in Z} V$ is a Prüfer domain with torsion Picard group and quotient field $F$ if and only if for each A-morphism $\phi: Z \rightarrow \mathbb{P}_{A}^{1}$ there is a homogeneous polynomial $f \in A\left[T_{0}, T_{1}\right]$ of positive degree such that the image of $\phi$ is in $\left(\mathbb{P}_{A}^{1}\right)_{f}$.

Proof. The choice of the subring $D$ of $F$ was arbitrary, so for the sake of this proof we may assume without loss of generality that $D=A$ and apply then the preceding results to $A$. Suppose that for each $A$-morphism $\phi: Z \rightarrow \mathbb{P}_{A}^{1}$ there exists a homogeneous polynomial $f \in A\left[T_{0}, T_{1}\right]$ of positive degree such that the image of $\phi$ is in the affine subset $\left(\mathbb{P}_{A}^{1}\right)_{f}$. By Theorem 3.1, $A$ is a Prüfer domain with quotient field $F$. Thus, to prove that $A$ has torsion Picard group, it suffices to show that for each two-generated ideal $\left(t_{0}, t_{1}\right) A$ of $A$, there exists $e>0$ such that $\left(t_{0}, t_{1}\right)^{e} A$ is a principal ideal (see, for example, the proof of Theorem 2.2 of [Gilmer 1969]). Let $t_{0}, t_{1} \in F$, and let $\phi: Z \rightarrow \mathbb{P}_{A}^{1}$ be the morphism defined by $t_{0}, t_{1}$. Then by assumption, there exists a homogeneous polynomial $f \in A\left[T_{0}, T_{1}\right]$ of positive degree $d$ such that the image of $Z$ in $\mathbb{P}_{A}^{1}$ is contained in $\left(\mathbb{P}_{A}^{1}\right)_{f}$. Thus by Lemma $3.4,\left(t_{0}, t_{1}\right)^{d} A$ is a principal ideal. 
Conversely, let $\phi: Z \rightarrow \mathbb{P}_{A}^{1}$ be an $A$-morphism. Then by Proposition 2.3, there exist $t_{0}, t_{1} \in F$ such that $\phi$ is defined by $t_{0}, t_{1}$. Since $A$ has torsion Picard group and quotient field $F$, there exists $d>0$ such that $\left(t_{0}, t_{1}\right)^{d} A=u A$ for some $u \in\left(t_{0}, t_{1}\right)^{d} A$. Since $u$ is an element of $\left(t_{0}, t_{1}\right)^{d} A$, there exists a homogeneous polynomial $f \in$ $A\left[T_{0}, T_{1}\right]$ of degree $d$ such that $f\left(t_{0}, t_{1}\right)=u$, and hence by Lemma 3.4, the image of the morphism $\phi$ is contained in $\left(\mathbb{P}_{A}^{1}\right)_{f}$.

For applications such as those discussed in (2) and (3) of the introduction, one needs to work with $D$-morphisms into the projective line over $D$, rather than $A$. This involves a change of base, but causes no difficulties when verifying that $A$ is a Prüfer domain. However, the converse of Theorem 3.5 (which is not needed in the applications in (2) and (3) of the introduction) is lost in the base change.

Corollary 3.6. If for each $D$-morphism $\phi: Z \rightarrow \mathbb{P}_{D}^{1}$ there exists a homogeneous polynomial $f \in D\left[T_{0}, T_{1}\right]$ of positive degree such that the image of $Z$ is contained in $\left(\mathbb{P}_{D}^{1}\right)_{f}$, then $A$ is a Prüfer domain with torsion Picard group and quotient field $F$. Proof. Let $\phi: Z \rightarrow \mathbb{P}_{A}^{1}$ be a $D$-morphism, and let $\alpha: \mathbb{P}_{A}^{1} \rightarrow \mathbb{P}_{D}^{1}$ be the change of base morphism. By assumption, there exists a homogeneous polynomial $f \in D\left[T_{0}, T_{1}\right]$ such that the image of $\alpha \circ \phi$ is contained in $\left(\mathbb{P}_{D}^{1}\right)_{f}$. Then the image of $\phi$ is contained in $\left(\mathbb{P}_{A}^{1}\right)_{f}$, and the corollary follows from Theorem 3.1.

Let $n$ be a positive integer. An abelian group $G$ is an $n$-group if each element of $G$ has finite order and this order is divisible by only such primes that also appear as factors of $n$. If $A$ is a Prüfer domain with quotient field $F$, then the Picard group of $A$ is an $n$-group if and only if for each $t \in F$ there exists $k>0$ such that $(A+t A)^{n^{k}}$ is a principal fractional ideal of $A$ [Roquette 1973, Lemma 1].

Remark 3.7. If each homogeneous polynomial $f$ arising as in the statement of the corollary can be chosen with degree $\leq n$ ( $n$ fixed), then the Picard group of the Prüfer domain $A$ is an $n$-group. For when $t \in F$ and $\phi: Z \rightarrow \mathbb{P}_{D}^{1}$ is the $D$-morphism defined by $1, t$, then with $f$ the polynomial of degree $\leq n$ given by the corollary, Lemma 3.4 shows that $(A+t A)^{n}$ is a principal fractional ideal of $A$. In particular, when for each $D$-morphism $\phi: Z \rightarrow \mathbb{P}_{D}^{1}$ there exists a linear homogeneous polynomial $f \in A\left[T_{0}, T_{1}\right]$ such that the image of $\phi$ is contained in $\left(\mathbb{P}_{A}^{1}\right)_{f}$, the ring $A$ is a Bézout domain with quotient field $F$.

The next corollary is a stronger version of statement (4) in the introduction.

Corollary 3.8. If $D$ is a local domain and $Z$ has cardinality less than that of the residue field of $D$, then $A$ is a Bézout domain with quotient field $F$.

Proof. Let $\phi: Z \rightarrow \mathbb{P}_{D}^{1}$ be a $D$-morphism. For each $P \in \operatorname{Proj}\left(D\left[T_{0}, T_{1}\right]\right)$, let $\Delta_{P}=\left\{d \in D: T_{0}+d T_{1} \in P\right\}$. Then all the elements of $\Delta_{P}$ have the same image in the residue field of $D$. Indeed, if $d_{1}, d_{2} \in \Delta_{P}$, then 


$$
\left(d_{1}-d_{2}\right) T_{1}=\left(T_{0}+d_{1} T_{1}\right)-\left(T_{0}+d_{2} T_{1}\right) \in P .
$$

If $T_{1} \in P$, then since $T_{0}+d_{1} T_{1} \in P$, this forces $\left(T_{0}, T_{1}\right) \subseteq P$, a contradiction to the fact that $P \in \operatorname{Proj}\left(D\left[T_{0}, T_{1}\right]\right)$. Therefore, $T_{1} \notin P$, so that $d_{1}-d_{2} \in P \cap D \subseteq \mathfrak{m}:=$ maximal ideal of $D$, which shows that all the elements of $\Delta_{P}$ have the same image in the residue field of $D$. Let $X$ denote the image of $\phi$ in $\mathbb{P}_{D}^{1}$. Then since $|X|<|D / \mathfrak{m}|$, there exists $d \in D \backslash \bigcup_{P \in X} \Delta_{P}$, and hence $f\left(T_{0}, T_{1}\right):=T_{0}+d T_{1} \notin P$ for all $P \in X$. Thus the image of $\phi$ is in $\left(\mathbb{P}_{D}^{1}\right)_{f}$, and by Corollary 3.6 and Remark 3.7, $A$ is a Bézout domain with quotient field $F$.

The following corollary is a small improvement of a theorem of Rush [2001, Theorem 1.4]. Whereas the theorem of Rush requires that $1, t, t^{2}, \ldots, t^{d_{t}} \in f_{t}(t) A$, we need only that $1, t^{d_{t}} \in f_{t}(t) A$.

Corollary 3.9. The ring $A$ is a Prüfer domain with torsion Picard group and quotient field $F$ if and only if for each $0 \neq t \in F$, there is a polynomial $f_{t}(T) \in A[T]$ of positive degree $d_{t}$ such that $1, t^{d_{t}} \in f_{t}(t) A$.

Proof. If $A$ is a Prüfer domain with torsion Picard group and quotient field $F$, then for each $0 \neq t \in F$, there is $d_{t}>0$ such that $(1, t)^{d_{t}} A$ is a principal fractional ideal of $A$. Since $A$ is a Prüfer domain, local verification shows that $(1, t)^{d_{t}} A=\left(1, t^{d_{t}}\right) A$, and it follows that there is a polynomial $f_{t}(T) \in A[T]$ of positive degree $d_{t}$ such that $1, t^{d_{t}} \in f_{t}(t) A$.

To prove the converse, we use Theorem 3.5. Let $\phi: Z \rightarrow \mathbb{P}_{D}^{1}$ be a $D$-morphism. Then by Proposition 2.3, there exists $0 \neq t \in F$ such that $\phi$ is defined by $1, t$. By assumption, there is a polynomial $f_{t}(T) \in A[T]$ of positive degree $d_{t}$ such that $1, t^{d_{t}} \in f_{t}(t) A$. Set $g_{t}\left(T_{0}, T_{1}\right)=f_{t}\left(T_{0} / T_{1}\right) T_{1}^{d_{t}}$, so that $g_{t}\left(T_{0}, T_{1}\right)$ is a homogeneous form of positive degree. Then $1, t^{d_{t}} \in g_{t}(t, 1) A$, and by Lemma 3.4, the image of $\phi$ is in $\left(\mathbb{P}_{A}^{1}\right)_{g}$. By Theorem 3.5, $A$ is a Prüfer domain with torsion Picard group and quotient field $F$.

Rush [2001, Theorem 2.2] proves that when $f$ is a monic polynomial of positive degree in $A[T]$, then (a) $\{1 / f(t): t \in F\} \subseteq A$ if and only if (b) the image of $f$ in $\left(V / \mathfrak{M}_{V}\right)[T]$ has no root in $V / \mathfrak{M}_{V}$ for each $V \in Z$ if and only if (c) $A$ is a Prüfer domain and $f(a)$ is a unit in $A$ for each $a \in A$. As Rush points out, Gilmer's theorem discussed in (2) of the introduction follows quickly from the equivalence of (a) and (b) and Corollary 3.9; see the discussion on pp. 314-315 of [Rush 2001]. Similarly, the results of Loper and Roquette described in (3) of the introduction also follow from Corollary 3.9 and the equivalence of (a) and (b). Thus all the constructions in (1)-(4) of the introduction are recovered by the results in this section. 


\section{The case where $D$ is a local ring}

This section focuses on the case where $D$ is a local ring that is integrally closed in $F$. (By a local ring, we mean a ring that has a unique maximal ideal; in particular, we do not require local rings to be Noetherian.) In such a case, as is noted in the proof of Theorem 4.2, every proper subset of closed points of $\mathbb{P}_{D}^{1}$ is contained in an affine open subset of $\mathbb{P}_{D}^{1}$, a fact which leads to a stronger result than could be obtained in the last section. To prove the theorem, we need a coset version of homogeneous prime avoidance. The proof of the lemma follows Gabber, Liu and Lorenzini [Gabber et al. 2013] but involves a slight modification to permit cosets.

Lemma 4.1. (cf. [Gabber et al. 2013, Lemma 4.11]) Let $R=\bigoplus_{i=0}^{\infty} R_{i}$ be a graded ring, and let $P_{1}, \ldots, P_{n}$ be incomparable homogeneous prime ideals not containing $R_{1}$. Let $I=\bigoplus_{i=0}^{\infty} I_{i}$ be a homogeneous ideal of $R$ such that $I \nsubseteq P_{i}$ for each $i=1, \ldots, n$. Then there exists $e_{0}>0$ such that for all $e \geq e_{0}$ and $r_{1}, \ldots, r_{n} \in R$, $I_{e} \nsubseteq \bigcup_{i=1}^{n}\left(P_{i}+r_{i}\right)$.

Proof. The proof is by induction on $n$. For the case $n=1$, let $s$ be a homogeneous element in $I \backslash P_{1}$, let $e_{0}=\operatorname{deg} s$, let $e \geq e_{0}$ and let $t \in R_{1} \backslash P_{1}$. Suppose that $r_{1} \in R$ and $I_{e} \subseteq P_{1}+r_{1}$. Then since $0 \in I_{e}$, this forces $r_{1} \in P_{1}$ and hence $s t^{e-e_{0}} \in I_{e} \subseteq P_{1}$, a contradiction to the fact that neither $s$ nor $t$ is in $P_{1}$. Thus $I_{e} \nsubseteq P_{1}+r_{1}$. Next, let $n>1$, and suppose that the lemma holds for $n-1$. Then since the $P_{i}$ are incomparable, $I P_{1} \ldots P_{n-1} \nsubseteq P_{n}$, and by the case $n=1$, there exists $f_{0}>0$ such that for all $f \geq f_{0}$ and $r_{n} \in R$ we have $\left(I P_{1} \ldots P_{n-1}\right)_{f} \not \subset\left(P_{n}+r_{n}\right)$. Also, by the induction hypothesis, there exists $g_{0}>0$ such that for all $g \geq g_{0}$ and $r_{1}, \ldots, r_{n-1} \in R$ we have $\left(I P_{n}\right)_{g} \nsubseteq \bigcup_{i=1}^{n-1}\left(P_{i}+r_{i}\right)$. Let $e_{0}=\max \left\{f_{0}, g_{0}\right\}$, let $e \geq e_{0}$ and let $r_{1}, \ldots, r_{n} \in R$. Then in light of the above considerations, we may choose $a \in\left(I P_{1} \ldots P_{n-1}\right)_{e} \backslash\left(P_{n}+r_{n}\right)$ and $b \in\left(I P_{n}\right)_{e} \backslash \bigcup_{i=1}^{n-1}\left(P_{i}+r_{i}\right)$. Then $a+b \in I_{e} \backslash \bigcup_{i=1}^{n}\left(P_{i}+r_{i}\right)$.

Theorem 4.2. Suppose $D$ is local and integrally closed in $F$ and only finitely many valuation rings in $Z$ do not dominate $D$. If no $D$-morphism $Z \rightarrow \mathbb{P}_{D}^{1}$ has every closed point of $\mathbb{P}_{D}^{1}$ in its image, then $A=\bigcap_{V \in Z} V$ is a Prüfer domain with torsion Picard group and quotient field $F$.

Proof. Let $S=D\left[T_{0}, T_{1}\right]$. By Corollary 3.6, it suffices to show that for each $D$-morphism $\phi: Z \rightarrow \mathbb{P}_{D}^{1}$, there is a homogeneous polynomial $f \in S$ of positive degree such that the image of $\phi$ is in $\left(\mathbb{P}_{D}^{1}\right)_{f}$. To this end, let $\phi: Z \rightarrow \mathbb{P}_{D}^{1}$ be a $D$-morphism. By assumption, there is a closed point $x \in \mathbb{P}_{D}^{1}$ not in the image of $\phi$. Let $\pi: \mathbb{P}_{D}^{1} \rightarrow \operatorname{Spec}(D)$ be the structure morphism. Since $\pi$ is a proper morphism, $\pi$ is closed and hence $\pi(x)$ is a closed point in $\operatorname{Spec}(D)$. Thus since $D$ is local, $\pi(x)$ is the maximal ideal $\mathfrak{m}$ of $D$. Let $\mathbb{k}$ be the residue field of $D$. Then, with $Q$ the homogeneous prime ideal in $S$ corresponding to $x$, we must have $\mathfrak{m} \subseteq Q$, 
and hence Proj $\left(\mathbb{k}\left[T_{0}, T_{1}\right]\right)$ is isomorphic to a closed subset of $\mathbb{P}_{D}^{1}$ containing $Q$. Since a homogeneous prime ideal in $\operatorname{Proj}\left(\mathbb{k}\left[T_{0}, T_{1}\right]\right)$ is generated by a homogeneous polynomial in $\mathbb{k}\left[T_{0}, T_{1}\right]$, it follows that there is a homogeneous polynomial $g \in S$ of positive degree $d$ such that $Q=(\mathfrak{m}, g) S$. Since, as noted above, every prime ideal in $\mathbb{P}_{D}^{1}=\operatorname{Proj}(S)$ corresponding to a closed point in $\mathbb{P}_{D}^{1}$ contains $\mathfrak{m}$, it follows that every closed point in $\mathbb{P}_{D}^{1}$ distinct from $x$ is contained in $\left(\mathbb{P}_{D}^{1}\right)_{g}$. Thus if every valuation ring in $Z$ other than $F$ dominates $D$, then the image of $\phi$ is contained in $\left(\mathbb{P}_{D}^{1}\right)_{g}$, which proves the theorem.

It remains to consider the case where $Z$ also contains, in addition to the valuation ring $F$, valuation rings $V_{1}, \ldots, V_{n}$ that are not centered on the maximal ideal $\mathfrak{m}$ of $D$. Let $P_{1}, \ldots, P_{n}$ be the homogeneous prime ideals of $S$ that are the images under $\phi$ of $V_{1}, \ldots, V_{n}$, respectively. Let $I=\mathfrak{m} S$. No $V_{i}$ dominates $D$, so since $\phi$ is a morphism of locally ringed spaces, $I \nsubseteq P_{i}$ for all $i=1, \ldots, n$. We may assume $P_{1}, \ldots, P_{k}$ are the prime ideals that are maximal in the set $\left\{P_{1}, \ldots, P_{n}\right\}$. Then by Lemma 4.1, there exists $e>0$ such that $I_{d e} \nsubseteq \bigcup_{i=1}^{k}\left(P_{i}+g^{e}\right)$. Let $h$ be a homogeneous element in $I_{d e} \backslash \bigcup_{i=1}^{k}\left(P_{i}+g^{e}\right)$. Since $P_{1}, \ldots, P_{k}$ are maximal in $\left\{P_{1}, \ldots, P_{n}\right\}$, it follows that $h \in I_{d e} \backslash \bigcup_{i=1}^{n}\left(P_{i}+g^{e}\right)$. Set $f=h-g^{e}$. Then $f \notin P_{i}$ for all $i$. In particular, $f \neq 0$, and hence $f$ is homogeneous of degree $d e$. Since $f \notin P_{1} \cup \cdots \cup P_{n}$, we have $P_{1}, \ldots, P_{n} \in\left(\mathbb{P}_{D}^{1}\right)_{f}$.

Finally we show that every closed point of $\mathbb{P}_{D}^{1}$ distinct from $x$ is in $\left(\mathbb{P}_{D}^{1}\right)_{f}$. Let $L$ be a prime ideal in $\operatorname{Proj}(S)$ corresponding to a closed point distinct from $x$. Then $L \neq Q$, and to finish the proof, we need only show that $f \notin L$. As noted above, $\mathfrak{m} \subseteq L$, so if $f \in L$, then since $h \in \mathfrak{m} S$, we have $g^{e} \in L$. But then $Q=(\mathfrak{m}, g) S \subseteq L$, forcing $Q=L$ since $Q$ is maximal in $\operatorname{Proj}(S)$. This contradiction implies that $f \notin L$, and hence every closed point of $\mathbb{P}_{D}^{1}$ distinct from $x$ is in $\left(\mathbb{P}_{D}^{1}\right)_{f}$, which completes the proof.

Remark 4.3. When the valuation rings in $Z$ do not dominate $D$, the theorem can still be applied if there exists $Y \subseteq \mathfrak{X}$ containing $F$ such that (a) each valuation ring in $Y$ other than $F$ dominates $D$, (b) each valuation ring in $Z$ specializes to a valuation ring in $Y$, and (c) no $D$-morphism $\phi: Y \rightarrow \mathbb{P}_{D}^{1}$ has every closed point in its image. For by the theorem, the holomorphy ring of $Y$ is a Prüfer domain with torsion Picard group and quotient field $F$. As an overring of the holomorphy ring of $Y$, the holomorphy ring of $Z$ has these same properties also.

The following corollary shows how the theorem can be used to prove that real holomorphy rings can be intersected with finitely many nondominating valuation rings and the result remains a Prüfer domain with quotient field $F$. In general an intersection of a Prüfer domain and a valuation domain need not be a Prüfer domain. For example, when $D$ is a two-dimensional local Noetherian UFD with quotient field $F$ and $f$ is an irreducible element of $D$, then $D_{f}$ is a PID and $D_{(f)}$ 
is a valuation ring, but $D=D_{f} \cap D_{(f)}$, so that the intersection is not Prüfer. This example can be modified to show more generally that for this choice of $D$, there exist quasicompact schemes in $\mathfrak{X}$ that are not affine.

Corollary 4.4. Suppose D is essentially of finite type over a real-closed field and that $F$ and the residue field of $D$ are formally real. Let $H$ be the real holomorphy ring of $F / D$. Then for any valuation rings $V_{1}, \ldots, V_{n} \in \mathfrak{X}$ not dominating $D$, the ring $H \cap V_{1} \cap \cdots \cap V_{n}$ is a Prüfer domain with torsion Picard group and quotient field $F$.

Proof. Each formally real valuation ring in $\mathfrak{X}$ specializes to a formally real valuation ring dominating $D$ (this can be deduced, for example, from Theorem 23 of [Kuhlmann 2004]). Let $Y$ be the set of all the formally real valuation rings dominating $D$, let $Z=Y \cup\left\{F, V_{1}, \ldots, V_{n}\right\}$, and let $\phi: Z \rightarrow \mathbb{P}_{D}^{1}$ be a $D$-morphism. Then the image of $Y$ under $\phi$ is contained in $\left(\mathbb{P}_{D}^{1}\right)_{f}$, where $f\left(T_{0}, T_{1}\right)=T_{0}^{2}+T_{1}^{2}$. Because $V_{1}, \ldots, V_{n}$ do not dominate $D$, they are not mapped by $\phi$ to closed points of $\mathbb{P}_{D}^{1}$. Thus the corollary follows from Theorem 4.2.

We include the last corollary as more of a curiosity than an application. Suppose that $D$ has quotient field $F$. A valuation ring $V$ in $\mathfrak{X}$ admits local uniformization if there exists a projective model $X$ of $F / D$ such that $V$ dominates a regular local ring in $X$. Thus if $\operatorname{Spec}(D)$ has a resolution of singularities, then every valuation ring in $\mathfrak{X}$ admits local uniformization. If $D$ is essentially of finite type over a field $k$ of characteristic 0 , then $D$ has a resolution of singularities by the theorem of Hironaka, but when $k$ has positive characteristic, it is not known in general whether local uniformization holds in dimension greater than 3; see, for example, [Cutkosky 2004; Temkin 2013].

Corollary 4.5. Suppose that $D$ is a quasiexcellent integrally closed local Noetherian domain with quotient field $F$. If there exists a valuation ring in $\mathfrak{X}$ that dominates $D$ but does not admit local uniformization, and $Y$ consists of all such valuation rings, then the holomorphy ring of $Y$ is a Prüfer domain with torsion Picard group. Proof. Let $Z=Y \cup\{F\}$, and let $\phi: Z \rightarrow \mathbb{P}_{D}^{1}$ be a $D$-morphism. Then by Proposition 2.3, $\phi$ factors through a projective model $X$ of $F / D$. Since $Y$ is nonempty, the projective model $X$ has a singularity, and thus since $D$ is quasiexcellent, the singular points of $X$ are contained in a proper nonempty closed subset of $X$. In particular, there are closed points of $X$ that are not in the image of the domination map $Z \rightarrow X$, and hence there are closed points of $\mathbb{P}_{D}^{1}$ that are not in the image of $\phi$. Therefore, by Theorem 4.2, $A$ is a Prüfer domain with torsion Picard group and quotient field $F$.

In particular, all the valuation rings that dominate $D$ and do not admit local uniformization lie in an affine scheme in $\mathfrak{X}$. 


\section{References}

[Becker 1982] E. Becker, "The real holomorphy ring and sums of $2 n$-th powers", pp. 139-181 in Géométrie algébrique réelle et formes quadratiques (Rennes, 1981), edited by J.-L. Colliot-Thèléne et al., Lecture Notes in Math. 959, Springer, Berlin, 1982. MR 84g:12032 Zbl 0508.12020

[Bruns and Herzog 1993] W. Bruns and J. Herzog, Cohen-Macaulay rings, Cambridge Studies in Advanced Mathematics 39, Cambridge University Press, 1993. MR 95h:13020 Zbl 0788.13005

[Cutkosky 2004] S. D. Cutkosky, Resolution of singularities, Graduate Studies in Mathematics 63, American Mathematical Society, Providence, RI, 2004. MR 2005d:14022 Zbl 1076.14005

[Dress 1965] A. Dress, "Lotschnittebenen mit halbierbarem rechtem Winkel", Arch. Math. (Basel) 16 (1965), 388-392. MR 33 \#1781 Zbl 0137.40204

[Eisenbud and Harris 2000] D. Eisenbud and J. Harris, The geometry of schemes, Graduate Texts in Mathematics 197, Springer, New York, 2000. MR 2001d:14002 Zbl 0960.14002

[Fontana et al. 1997] M. Fontana, J. A. Huckaba, and I. J. Papick, Prüfer domains, Monographs and Textbooks in Pure and Applied Mathematics 203, Marcel Dekker, New York, 1997. MR 98d:13021 Zbl 0861.13006

[Fuchs and Salce 2001] L. Fuchs and L. Salce, Modules over non-Noetherian domains, Mathematical Surveys and Monographs 84, American Mathematical Society, Providence, RI, 2001. MR 2001i:13002 Zbl 0973.13001

[Gabber et al. 2013] O. Gabber, Q. Liu, and D. Lorenzini, "The index of an algebraic variety", Invent. Math. 192:3 (2013), 567-626. MR 3049930 Zbl 1268.13009

[Gilmer 1968] R. W. Gilmer, Multiplicative ideal theory, Queen's Papers in Pure and Applied Mathematics 12, Queen's University, Kingston, ON, 1968. MR 37 \#5198 Zbl 0155.36402

[Gilmer 1969] R. W. Gilmer, "Two constructions of Prüfer domains", J. Reine Angew. Math. 239-240 (1969), 153-162. MR 41 \#1710 Zbl 0184.29102

[Hartshorne 1977] R. Hartshorne, Algebraic geometry, Graduate Texts in Mathematics 52, Springer, New York, 1977. MR 57 \#3116 Zbl 0367.14001

[Holme 2012] A. Holme, A royal road to algebraic geometry, Springer, Berlin, 2012. MR 2858123 Zbl 1237.14001

[de Jong et al. 2005-] A. J. de Jong et al., "The Stacks project", electronic reference, 2005-, http:// stacks.math.columbia.edu.

[Knebusch and Zhang 2002] M. Knebusch and D. Zhang, Manis valuations and Prüfer extensions, I: A new chapter in commutative algebra, Lecture Notes in Math. 1791, Springer, Berlin, 2002. MR 2003j:13002 Zbl 1033.13001

[Kuhlmann 2004] F.-V. Kuhlmann, "Places of algebraic function fields in arbitrary characteristic", Adv. Math. 188:2 (2004), 399-424. MR 2005h:12014 Zbl 1134.12304

[Larsen and McCarthy 1971] M. D. Larsen and P. J. McCarthy, Multiplicative theory of ideals, Pure and Applied Mathematics 43, Academic Press, New York, 1971. MR 54 \#2629 Zbl 0237.13002

[Loper 1994] K. A. Loper, “On Prüfer non-D-rings”, J. Pure Appl. Algebra 96:3 (1994), 271-278. MR 95k:13024 Zbl 0840.13009

[Matsumura 1980] H. Matsumura, Commutative ring theory, Kyoritsu Shuppan, Tokyo, 1980. In Japanese; translated in Cambridge Studies in Advanced Mathematics 8, Cambridge University Press, 1986. 2nd ed. in 1989. MR 90i:13001 Zbl 0603.13001

[Nagata 1962] M. Nagata, Local rings, Interscience Tracts in Pure and Applied Mathematics 13, Wiley, New York, 1962. MR 27 \#5790 Zbl 0123.03402 
[Olberding 2006] B. Olberding, "Holomorphy rings of function fields", pp. 331-347 in Multiplicative ideal theory in commutative algebra, edited by J. W. Brewer et al., Springer, New York, 2006. MR 2008f:13035 Zbl 1118.13022

[Olberding 2007] B. Olberding, "Irredundant intersections of valuation overrings of two-dimensional Noetherian domains”, J. Algebra 318:2 (2007), 834-855. MR 2009b:13057 Zbl 1137.13003

[Olberding 2008] B. Olberding, "Overrings of two-dimensional Noetherian domains representable by Noetherian spaces of valuation rings", J. Pure Appl. Algebra 212:7 (2008), 1797-1821. MR 2009c: 13008 Zbl 1140.13006

[Olberding 2011] B. Olberding, "Intersections of valuation overrings of two-dimensional Noetherian domains", pp. 335-361 in Commutative algebra: Noetherian and non-Noetherian perspectives, edited by M. Fontana et al., Springer, New York, 2011. MR 2012b:13014 Zbl 1225.13010

[Olberding and Roitman 2006] B. Olberding and M. Roitman, "The minimal number of generators of an invertible ideal", pp. 349-367 in Multiplicative ideal theory in commutative algebra, edited by J. W. Brewer et al., Springer, New York, 2006. MR 2007i:13021 Zbl 1118.13019

[Roquette 1973] P. Roquette, "Principal ideal theorems for holomorphy rings in fields", J. Reine Angew. Math. 262-263 (1973), 361-374. MR 48 \#3950 Zbl 0271.13009

[Rush 2001] D. E. Rush, "Bezout domains with stable range 1", J. Pure Appl. Algebra 158:2-3 (2001), 309-324. MR 2002k:13034 Zbl 1094.13531

[Schülting 1979] H.-W. Schülting, "Über die Erzeugendenanzahl invertierbarer Ideale in Prüferringen”, Comm. Algebra 7:13 (1979), 1331-1349. MR 81j:12016 Zbl 0432.13010

[Schülting 1982] H.-W. Schülting, "Real holomorphy rings in real algebraic geometry", pp. 433-442 in Géométrie algébrique réelle et formes quadratiques (Rennes, 1981), edited by J.-L. ColliotThèléne et al., Lecture Notes in Math. 959, Springer, Berlin, 1982. MR 84d:14016 Zbl 0498.14013

[Swan 1984] R. G. Swan, "n-generator ideals in Prüfer domains”, Pacific J. Math. 111:2 (1984), 433-446. MR 85k:13019 Zbl 0546.13008

[Temkin 2013] M. Temkin, "Inseparable local uniformization”, J. Algebra 373 (2013), 65-119. MR 2995017 Zbl 1276.14021

[Temkin and Tyomkin 2013] M. Temkin and I. Tyomkin, "Prüfer algebraic spaces", preprint, 2013. arXiv 1101.3199

[Zariski and Samuel 1975] O. Zariski and P. Samuel, Commutative algebra, vol. II, Graduate Texts in Mathematics 29, Springer, New York, 1975. MR 52 \#10706 Zbl 0313.13001

Received January 22, 2014. Revised July 26, 2014.

\section{BRUCE OLBERDING}

DEPARTMENT OF MATHEMATICAL SCIENCES

NeW MEXico State UniVERSity

LAS CRUCES, NM 88003-8001

UNITED STATES

olberdin@nmsu.edu 


\title{
PACIFIC JOURNAL OF MATHEMATICS
}

\author{
msp.org/pjm
}

Founded in 1951 by E. F. Beckenbach (1906-1982) and F. Wolf (1904-1989)

\section{EDITORS}

Don Blasius (Managing Editor)

Department of Mathematics

University of California

Los Angeles, CA 90095-1555

blasius@math.ucla.edu

\author{
Paul Balmer \\ Department of Mathematics \\ University of California \\ Los Angeles, CA 90095-1555 \\ balmer@math.ucla.edu \\ Robert Finn \\ Department of Mathematics \\ Stanford University \\ Stanford, CA 94305-2125 \\ finn@math.stanford.edu \\ Sorin Popa \\ Department of Mathematics \\ University of California \\ Los Angeles, CA 90095-1555 \\ popa@math.ucla.edu
}

\author{
Vyjayanthi Chari \\ Department of Mathematics \\ University of California \\ Riverside, CA 92521-0135 \\ chari@math.ucr.edu \\ Kefeng Liu \\ Department of Mathematics \\ University of California \\ Los Angeles, CA 90095-1555 \\ liu@math.ucla.edu \\ Jie Qing \\ Department of Mathematics \\ University of California \\ Santa Cruz, CA 95064 \\ qing@ cats.ucsc.edu
}

\section{PRODUCTION}

Silvio Levy, Scientific Editor, production@msp.org

\section{SUPPORTING INSTITUTIONS}

ACADEMIA SINICA, TAIPEI

CALIFORNIA INST. OF TECHNOLOGY

INST. DE MATEMÁTICA PURA E APLICADA

KEIO UNIVERSITY

MATH. SCIENCES RESEARCH INSTITUTE

NEW MEXICO STATE UNIV.

OREGON STATE UNIV.

\author{
STANFORD UNIVERSITY \\ UNIV. OF BRITISH COLUMBIA \\ UNIV. OF CALIFORNIA, BERKELEY \\ UNIV. OF CALIFORNIA, DAVIS \\ UNIV. OF CALIFORNIA, LOS ANGELES \\ UNIV. OF CALIFORNIA, RIVERSIDE \\ UNIV. OF CALIFORNIA, SAN DIEGO \\ UNIV. OF CALIF., SANTA BARBARA
}

\author{
Daryl Cooper \\ Department of Mathematics \\ University of California \\ Santa Barbara, CA 93106-3080 \\ cooper@math.ucsb.edu \\ Jiang-Hua Lu \\ Department of Mathematics \\ The University of Hong Kong \\ Pokfulam Rd., Hong Kong \\ jhlu@maths.hku.hk \\ Paul Yang \\ Department of Mathematics \\ Princeton University \\ Princeton NJ 08544-1000 \\ yang@math.princeton.edu
}

These supporting institutions contribute to the cost of publication of this Journal, but they are not owners or publishers and have no responsibility for its contents or policies.

See inside back cover or msp.org/pjm for submission instructions.

The subscription price for 2015 is US \$420/year for the electronic version, and \$570/year for print and electronic.

Subscriptions, requests for back issues and changes of subscribers address should be sent to Pacific Journal of Mathematics, P.O. Box 4163, Berkeley, CA 94704-0163, U.S.A. The Pacific Journal of Mathematics is indexed by Mathematical Reviews, Zentralblatt MATH, PASCAL CNRS Index, Referativnyi Zhurnal, Current Mathematical Publications and Web of Knowledge (Science Citation Index).

The Pacific Journal of Mathematics (ISSN 0030-8730) at the University of California, c/o Department of Mathematics, 798 Evans Hall \#3840, Berkeley, CA 94720-3840, is published twelve times a year. Periodical rate postage paid at Berkeley, CA 94704, and additional mailing offices. POSTMASTER: send address changes to Pacific Journal of Mathematics, P.O. Box 4163, Berkeley, CA 94704-0163.

PJM peer review and production are managed by EditFLOW ${ }^{\circledR}$ from Mathematical Sciences Publishers.

\section{PUBLISHED BY}

\section{mathematical sciences publishers \\ nonprofit scientific publishing}

http://msp.org/

(C) 2015 Mathematical Sciences Publishers 


\section{PACIFIC JOURNAL OF MATHEMATICS}

Volume $273 \quad$ No. $2 \quad$ February 2015

Taut foliations in surface bundles with multiple boundary components

TEJAS KALELKAR and RACHEL ROBERTS

Some results on archimedean Rankin-Selberg integrals

JINGSONG CHAI

Prescribing the boundary geodesic curvature on a compact scalar-flat Riemann

307

surface via a flow method

\section{HONG ZHANG}

-1-Phenomena for the pluri $\chi_{y}$-genus and elliptic genus

PING LI

On the geometry of Prüfer intersections of valuation rings

BRUCE OLBERDING

Non-Kähler expanding Ricci solitons, Einstein metrics, and exotic cone structures

Maria Buzano, Andrew S. Dancer, Michael Gallaugher and MCKENZIE WANG

A note on $L$-packets and abelian varieties over local fields

JEFFREY D. ACHTER and CLIFTON CUNNINGHAM

Rotating drops with helicoidal symmetry

Bennett Palmer and Oscar M. Perdomo

The bidual of a radical operator algebra can be semisimple

Charles John READ

Dimension jumps in Bott-Chern and Aeppli cohomology groups

JIEZHU LIN and XUANMING YE

Fixed-point results and the Hyers-Ulam stability of linear equations of higher orders

BING XU, JANUSZ BRZDĘK and WEINIAN ZHANG

Complete curvature homogeneous metrics on $\mathrm{SL}_{2}(\mathbb{R})$ 